\title{
Diversidad, estructura y afinidades florísticas de un bosque temporalmente inundable de la Península de Yucatán
}

\author{
Cristopher Albor Pinto*, Juan Tun Garrido \& Juan Javier Ortiz Díaz \\ Departamento de Botánica, Facultad de Medicina Veterinaria y Zootecnia, Universidad Autónoma de Yucatán, km \\ 15.5 carretera Mérida-Xmatkuil, 97315, Mérida, Yucatán, México; cristopher.albor@posgrado.ecologia.edu.mx, \\ tgarrido@correo.uady.mx,odiaz@correo.uady.mx \\ * Correspondence
}

Recibido 09-IX-2016. Corregido 20-III-2017. Aceptado 18-IV-2017.

\begin{abstract}
Diversity, structure and floristic affinities of a temporarily flooded forest of the Yucatan Peninsula. Temporarily flooded forests (TFF's) are among the most endangered tree communities in Southeast Mexico and in a contradictory way among the least known in terms of their diversity. Therefore, the aim of this study was to characterize the richness, diversity (Shannon-Wiener) and dominance (relative importance value; RIV) of a TFF of the Yucatan Peninsula, and evaluate its floristic affinities with other TFF's and upland forests (UF's) of Southeast Mexico. A sampling method based on 10 quadrants of 10 x $20 \mathrm{~m}\left(2000 \mathrm{~m}^{2}\right)$ was used to record all woody individuals with diameter $\geq 1 \mathrm{~cm}$ and height $\geq 1.5 \mathrm{~m}$. A floristic similarity analysis was used including 192 genera of woody plants belonging to TFF's and UF's of the Yucatan and Tabasco TFF's. A Mantel correlation test was performed to determine if the geographic distance has effect on the floristic similarities between TFF's and UF's of the Yucatan peninsula. The TFF under study was characterized with a low level of diversity (2.79) and species richness (56), and a high dominance level (52.3\% of RIV in five species). Dominant species such as Haematoxylum campechianum, Dalbergia glabra, Cameraria latifolia, Byrsonima crassifolia, Crescentia cujete and Terminalia buceras showed a floristic neotropical affinity typical of floodable habitats. The structure of the community was characterized by an inverted " $\mathrm{J}$ " pattern for the distribution of individuals in the diametric and height classes. The high density of woody lianas, mainly of Dalbergia glabra (22\% of the recorded individuals) suggested an optimal state of conservation of the studied area. The floristic similarity analysis revealed that most of TFF's in Yucatan Peninsula were grouped with high similarity (55 \%) independently of the adjacent UF's; however, TFF's resulted similar among them (36\% similarity) when compared with TFF's from other regions ( $16 \%$ similarity). According to the Mantel test, the similarity between sites is not influenced by its geographical proximity $(r=-0.098, p>0.05)$. This emphasizes the need to generate conservation strategies for the TFF's of the Yucatan peninsula independently from other regions of Southeast Mexico. Our results suggest that the TFF of the studied region has a similar diversity from other TFF's in Southeast Mexico, a different floristic composition compared with adjacent UF's and a structure with an optimal conservation status, characteristics that indicate their importance for the regional flora conservation. Further ecological studies are needed to build a solid foundation for management and conservation for the TFF's. Rev. Biol. Trop. 65 (3): 868-880. Epub 2017 September 01.
\end{abstract}

Key words: low inundated forest, Yucatan peninsula, wetland, plant diversity, floristic relationships, Haematoxylum campechianum.

Los bosques temporalmente inundables (BTI's) son formaciones vegetales sujetas a inundaciones periódicas durante la estación lluviosa (Scott \& Carbonell, 1986; Finlayson \& van der Valk, 1995, 2012; Scott \& Jones, 1995; Berlanga-Robles, Ruiz-Luna, \& de la
Lanza Espino, 2008). En el sureste de México este tipo de humedales temporales son raros (Moreno-Casasola \& Infante-Mata, 2009), por lo que existen vacíos de conocimiento respecto a su distribución, composición florística, estructura y ecología (Lot \& Novelo, 1990; 
Maldonado-Sánchez et al., 2016). Esto es contrastante en relación a los bosques de tierras altas (BTA's), es decir, la gran mayoría de bosques que no sufren inundaciones, los cuales han sido ampliamente estudiados en temas de biodiversidad y conservación.

Los BTI's del sureste de México poseen diferentes orígenes, lo cual es de importancia porque determina su hidroperiodo (Moreno-Casasola, Infante-Mata, \& López-Rosas, 2012). Por ejemplo, zonas adyacentes a ríos como en la cuenca del sistema UsumacintaGrijalva, poseen altos niveles de inundación durante épocas de lluvias, mientras en planicies costeras como en Tamaulipas, Veracruz y Tabasco, presentan bajos niveles de inundación durante gran parte del año (Olmsted, 1993; Moreno-Casasola \& Infante-Mata, 2009). Los BTI's de la península de Yucatán son un caso particular, donde su origen no se relaciona a cuerpos de agua sino a "bajos inundables", es decir, zonas donde por desnivel del relieve, alta escorrentía y deficiencia de drenaje de suelos arcillosos, genera inundación durante la temporada de lluvias que persiste hasta por ocho meses (Miranda, 1978; Duch-Gary, 1989; Olmsted, 1993).

A diferencia de las especies de árboles que sobreviven en hábitats de inundación prolongada, en los BTI's, la mayoría carecen de adaptaciones morfológicas típicas de ambientes anóxicos (Kurzatkowski, Leuschner, \& Homeier, 2015), como son la presencia de contrafuertes, alta densidad de lenticelas, raíces zancudas o grandes diámetros (Parolin, De Simone, Haase, \& Waldhoff, 2004; López, 2009). En la península de Yucatán los arboles dominantes de BTI's son espinosos, muy ramificados, con alturas promedio de siete metros y caducifoleidad aproximada del $50 \%$ durante la época más seca del año (Miranda, 1978; Olmsted, 1993; Flores \& Espejel, 1994; Schultz, 2005). También, esta comunidad vegetal puede reconocerse por su desarrollo en suelos grises arcillosos tipo vertisol, con drenaje deficiente, localmente conocidos como Ak'alches (DuchGary, 1989; Palacio-Aponte, Noriega-Trejo, \& Zamora-Crescencio, 2002).
Los estudios florísticos-fisionómicos en la península de Yucatán refieren a los BTI's bajo diferentes nombres, a saber selva baja subperennifolia (Miranda, 1958, 1978; Miranda \& Hernández-X, 1963; Flores \& Espejel, 1994;), selva baja inundable (Olmsted \& Durán, 1986; Lot \& Novelo, 1990; Schultz, 2005), vegetación de bajos inundables (Lundell, 1934) y bosque espinoso (Rzedowski, 2006), aunque todos hacen alusión a la inundación temporal de hasta ocho meses, característica principal de esta formación vegetal.

La importancia ecológica de los BTI's es que son inundables y contribuyen con la riqueza de la flora regional y albergan especies raras (Flinn, Lechowicz, \& Waterway, 2008). Además, fungen como refugio de fauna en temporadas adversas (Briceño-Méndez, ReynaHurtado, Calmé, \& García-Gil, 2014) y proporcionan múltiples servicios ambientales como la captura de carbono (Moreno-Casasola \& Infante-Mata, 2009; Migeot \& Imbert, 2012). Los estudios ecológicos de los BTI’s en la Península de Yucatán abordan aspectos como su diversidad, estructura y composición de especies (Díaz-Gallegos, Castillo-Acosta, \& García-Gil, 2002; Hernández-Ramírez \& García-Méndez, 2015; Olmsted \& Durán, 1986), pero son aislados y carecen de información fitogeográfica y de conservación. Un estudio comparativo de los BTI`s de México permitirá valorar el papel que juegan en la conservación de la flora regional y el mantenimiento de la biodiversidad.

Hoy en día, la generación de información de los BTI's es prioritaria en la región debido al alto grado de transformación que sufren, a causa de diferentes impactos antrópicos. Son necesarios estudios que describan la diversidad y estructura de la comunidad, y que contribuyan a su conocimiento más profundo y apoyen futuras medidas para su conservación en la región. Por lo anterior, los objetivos de este estudio fueron: 1) caracterizar la diversidad y estructura de un BTI de la península de Yucatán, 2) describir las relaciones florísticas de los BTI's de la península de Yucatán con otros BTI's del sureste de México (Tabasco) y BTA's de la península de Yucatán. 


\section{MATERIALES Y MÉTODOS}

Área de estudio: El trabajo de campo se realizó de enero a marzo 2015, en los límites de la Reserva Estatal Biocultural del Puuc en el municipio de Tekax, al sur de Yucatán, México (Fig. 1; $19^{\circ} 56^{\prime} 11.27^{\prime \prime} \mathrm{N}-89^{\circ} 7^{\prime}$ 55.7" W). El clima predominantes es del tipo Aw1 (i ') g, cálido y húmedo con lluvias en verano, la temperatura media anual es de 26.3 ${ }^{\circ} \mathrm{C}$, con precipitación media anual de $1122 \mathrm{~mm}$ (INEGI, 2009). El tipo de vegetación dominante es el bosque tropical subcaducifolio, y en menor proporción se desarrollan sabanas y bosques temporalmente inundables (ZamoraCrescencio, García-Gil, Flores, \& Ortiz, 2008; Gutiérrez-Báez, Ortiz-Díaz, Flores-Guido \& Zamora-Crescencio, 2012).
En el área de estudio, los BTI's se distribuyen en aproximadamente el $2 \%$ de su superficie, en las llanuras de inundación kársticas (80 - $120 \mathrm{msnm}$ ) situadas bajo formaciones cerriles adyacentes (150 - 205 msnm). Ningún cuerpo de agua superficial (ríos o lagos) se relaciona a esta formación vegetal. Se desarrolla en suelos arcillosos grises tipo vertisol, con profundidad superior a $80 \mathrm{~cm}$, sin afloramientos rocosos, alta contractilidad en la estación seca y drenaje deficiente durante la temporada de lluvias (Bautista \& Zinck, 2010), conocidos localmente como "Ak'alches" o bajos inundables (Duch-Gary, 1988, 1989; Palacio-Aponte et al., 2002). Inundaciones irregulares de $0.1-1.5 \mathrm{~m}$ de profundidad pueden presentarse hasta por ocho meses al año (Flores \& Espejel, 1994; Olmsted \& Durán, 1986).

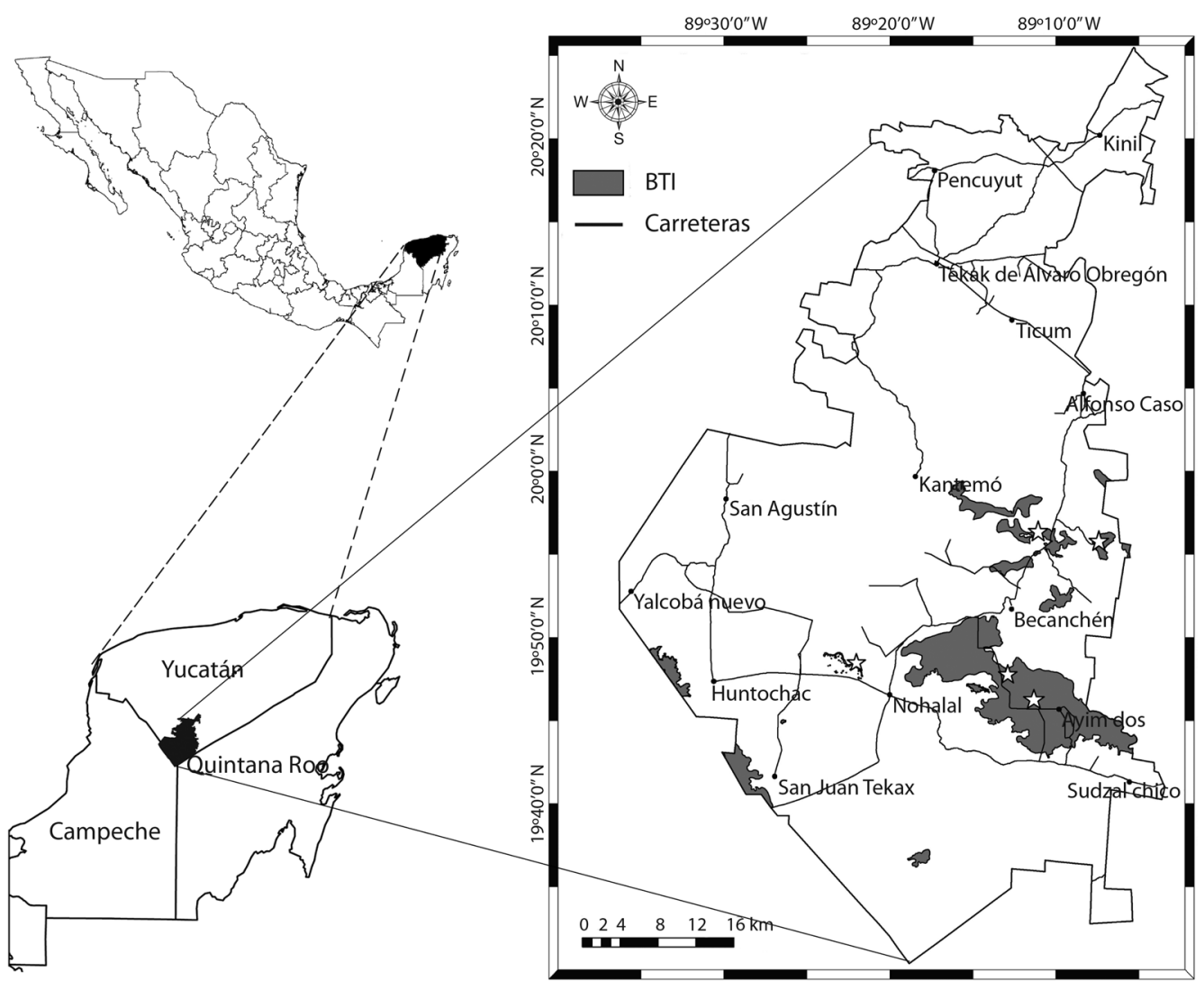

Fig. 1. Ubicación y delimitación del área de estudio. BTI= bosque temporalmente inundable. Sitios de muestreo señalados con estrellas.

Fig. 1. Location and delimitation of the study area. BTI= Temporarily flooded forests. Sample sites indicated with stars. 
Muestreo de la vegetación: Enclaves de BTI se identificaron mediante teledetección con el programa ArcGis 10.1. Se utilizaron como insumos imágenes Landsat ETM+ del 2010 e imágenes del Continuo de Elevaciones Mexicano (CEM 3.0). La distribución potencial del BTI fue verificada y corregida mediante recorridos en campo (Fig. 1), según las descripciones de este tipo de vegetación para la región (Miranda, 1958, 1978; Duch-Gary, 1989; Flores \& Espejel, 1994). Se seleccionaron cinco sitios de muestreo en zonas de BTI con buen estado de conservación (Fig. 1), es decir, aquellas áreas más arboladas y desarrolladas estructuralmente. Se mantuvo una distancia de separación mínima de $2 \mathrm{~km}$ entre sitios (16 \pm $7.5 \mathrm{~km}$ ). En cada sitio de muestreo, se establecieron dos parcelas de $10 \times 20 \mathrm{~m}\left(200 \mathrm{~m}^{2}\right)$, con lo que se obtuvo una superficie total de muestreo de $2000 \mathrm{~m}^{2}$ (0.2 ha), según la metodología propuesta por Gentry (1982). En cada parcela se midió el diámetro a la altura del pecho (estandarizada a $1.3 \mathrm{~m}$ ) a individuos leñosos con diámetro $\geq 1 \mathrm{~cm}$ y altura $\geq 1.5 \mathrm{~m}$. Los individuos se identificaron en campo, cuando esto no fue posible se recolectaron y utilizaron claves especializadas y comparación con especímenes del herbario de la Universidad Autónoma de Yucatán (UADY), así como con la base de datos Global Plants (ITHAKA, 2015). La identidad taxonómica se atribuyó de acuerdo al sistema de clasificación del Angiosperm Phylogeny Group III (APG III; Chase \& Reveal, 2009). Material de referencia de las especies fue depositado en los herbarios del Centro de Investigación Científica de Yucatán A. C. (CICY) y de la Universidad Autónoma de Yucatán (UADY) (Thiers, 2012).

Análisis de la diversidad, estructura y dominancia: Para evaluar la representatividad de la riqueza de especies obtenida con el esfuerzo de muestreo ( $0.2 \mathrm{ha})$, se estimó un intervalo de confianza al $95 \%$ y el estimador Chao 2 por su eficacia con datos de presencia-ausencia de las especies (Moreno \& Pineda, 2011), ambos calculados con el programa Estimate S versión 9.1.0 (Colwell, 2013). Se calculó la riqueza de especies e indicadores de diversidad $\alpha$ de Shannon-Wiener y equidad (Magurran, 1988). Se elaboraron histogramas de frecuencias para el reconocimiento de los patrones de distribución de las alturas y diámetros de los individuos registrados. La dominancia de las especies se calculó mediante el índice de Valor de Importancia Relativa (Mueller-Dombois \& Ellenberg, 1974) a partir de la suma de la frecuencia, densidad y dominancia relativas.

Análisis de similitud florística: Las afinidades florísticas del BTI de nuestra área de estudio se exploraron a través de un enfoque fenético con un análisis de conglomerados (UPGMA) mediante el coeficiente de similitud de Sorensen (Acosta, 2004; Giraldo-Cañas, 2001). Para ello se construyó una matriz de presencia-ausencia de los géneros de plantas leñosas presentes en estudios de BTI's de la península de Yucatán y del estado de Tabasco, y BTA's de la península de Yucatán (Cuadro 1). Estudios de BTI's del estado de Tabasco se incluyeron por su proximidad geográfica y afinidad florística con la Provincia Biótica de la península de Yucatán. El análisis se realizó mediante el paquete estadístico Multi-Variate Statistical Package (MVSP) versión 3.22.

Para determinar si la distancia geográfica entre sitios posee algún efecto sobre su similitud florística, se realizó una prueba de correlación de Mantel en el paquete estadístico XLSTAT Versión 2016.05.34059. Este análisis se realizó únicamente entre BTI's y BTA's de la península de Yucatán.

\section{RESULTADOS}

Análisis de diversidad, estructura y dominancia: Se registraron 1714 individuos pertenecientes a 56 especies, 43 géneros y 23 familias (Cuadro 2). Según el estimador de Chao 2, la curva de acumulación de especies no llegó a la asíntota (Fig. 2), sin embargo, se obtuvo una representación del $87.5 \%$ de la riqueza esperada. La riqueza de especies por parcela varió de 7 a $26(15 \pm 7)$. El valor 
CUADRO 1

Sitios incluidos en el análisis de similitud florística entre BTI’s y BTA’s de la península de Yucatán y Tabasco

TABLE 1

Sites included in the floristic similarity analysis between TFF's and UF's of the Yucatan peninsula and Tabasco

\begin{tabular}{|c|c|c|c|c|}
\hline No. & $\begin{array}{l}\text { Tipo de } \\
\text { bosque }\end{array}$ & Localidad & Clave & Referencia \\
\hline 1 & BTI & $\begin{array}{l}\text { Tekax, Yucatán } \\
\left(19^{\circ} 56{ }^{\prime} 11.27^{\prime}{ }^{\prime}, 89^{\circ} 7 ’ 55.71 ” O\right)\end{array}$ & 1. BTI, Tekax, Yuc. & Presente estudio \\
\hline 2 & BTI & $\begin{array}{l}\text { Reserva de la Biosfera de Calakmul } \\
\left(18^{\circ} 17^{\prime} 16^{\prime \prime} \mathrm{N}, 89^{\circ} 28^{\prime} 29^{\prime} \mathrm{O}\right)\end{array}$ & 2. BTI, Calakmul, Cam. & Díaz-Gallegos et al. (2002) \\
\hline 3 & BTI & $\begin{array}{l}\text { Reserva Ecológica El Edén } \\
\left(21^{\circ} 12^{\prime} 17.633^{\prime} \mathrm{N}, 87^{\circ} 10^{\prime} 35.59^{\prime} \mathrm{O}\right)\end{array}$ & 3. BTI, El Edén, Q. Roo & Schultz (2005) \\
\hline 4 & BTI & $\begin{array}{l}\text { Reserva de la Biosfera de Sian Ka'an } \\
\left(19^{\circ} 47^{\prime} 21.91^{\prime \prime N}, 87^{\circ} 45^{\prime} 7.40^{\prime \prime} \mathrm{O}\right)\end{array}$ & 4. BTI, Sian Ka'an, Q. Roo & Olmsted y Durán (1986) \\
\hline 5 & BTA & $\begin{array}{l}\text { Tekax, Yucatán } \\
\left(19^{\circ} 45^{\prime} 8.72 ” N, 89^{\circ} 16{ }^{\prime} 55.59^{\prime \prime} \mathrm{O}\right)\end{array}$ & 5. BTA, Tekax, Yuc. & Gutiérrez-Báez et al. (2012) \\
\hline 6 & BTA & $\begin{array}{l}\text { Reserva de la Biosfera de Calakmul } \\
\left(18^{\circ} 33^{\prime} 0.00^{\prime} \mathrm{N}, 89^{\circ} 55^{\prime} 39.00^{\prime \prime} \mathrm{O}\right)\end{array}$ & 6. BTA, Calakmul, Cam. & Chan-Dzul (2010) \\
\hline 7 & BTA & $\begin{array}{l}\text { Reserva Ecológica El Edén } \\
\left(21^{\circ} 13^{\prime} 57.12^{\prime} \mathrm{N}, 87^{\circ} 11^{\prime} 1.51^{\prime \prime} \mathrm{O}\right)\end{array}$ & 7. BTA, El Edén, Q. Roo & $\begin{array}{l}\text { Sánchez-Sánchez, Mendizábal- } \\
\text { Hernández y Calmé (2006) }\end{array}$ \\
\hline 8 & BTI & Centro, Tabasco (18 7’4”N, 9253’4”O) & 8. BTI, Centro, Tab. & Morales-Ramos (2008) \\
\hline 9 & BTI & $\begin{array}{l}\text { DACBiol, UJAT, Tabasco } \\
\left(17^{\circ} 59^{\prime} 26^{\prime \prime} \mathrm{N}, 92^{\circ} 58^{\prime} 16^{\prime \prime} \mathrm{O}\right)\end{array}$ & 9. BTI, UJAT, Tab. & Vázquez-Negrín et al. (2010) \\
\hline 10 & BTI & $\begin{array}{l}\text { Reserva Ecológica Yu-Balcah, Tabasco } \\
\left(17^{\circ} 35^{\prime} 56^{\prime} \mathrm{N}, 92^{\circ} 50^{\prime} 54^{\prime \prime} \mathrm{O}\right)\end{array}$ & 10. BTI, YCAH, Tab. & Maldonado-Sánchez et al. (2016) \\
\hline 11 & BTI & $\begin{array}{l}\text { Parque Estatal La Chontalpa, Tabasco } \\
\left(17^{\circ} 59^{\prime} 5^{\prime} \mathrm{N}, 93^{\circ} 34^{\prime} 13^{\prime \prime O}\right)\end{array}$ & 11. BTI, Chontalpa, Tab. & Maldonado-Sánchez et al. (2016) \\
\hline
\end{tabular}

\section{CUADRO 2}

Resumen taxonómico de las especies leñosas registradas en el área de estudio.

Familias ordenadas según su riqueza de especies

TABLE 2

Taxonomic summary of the woody species recorded in the study area. Families sorted by species richness

\begin{tabular}{lcclcc}
\multicolumn{1}{r}{ Familia } & Especies & Géneros & Familia & Especies & Géneros \\
Fabaceae & 14 & 11 & Sapindaceae & 2 & 2 \\
Rubiaceae & 5 & 2 & Apocynaceae & 1 & 1 \\
Euphorbiaceae & 3 & 2 & Combretaceae & 1 & 1 \\
Myrtaceae & 3 & 1 & Menispermaceae & 1 & 1 \\
Bignoniaceae & 3 & 3 & Malvaceae & 1 & 1 \\
Primulaceae & 3 & 2 & Celastraceae & 1 & 1 \\
Polygonaceae & 3 & 3 & Aristolochiaceae & 1 & 1 \\
Ebenaceae & 2 & 1 & Sapotaceae & 1 & 1 \\
Erythroxylaceae & 2 & 1 & Malpighiaceae & 1 & 1 \\
Salicaceae & 2 & 1 & Rhamnaceae & 1 & 1 \\
Capparaceae & 2 & 2 & Verbenaceae & 56 & 1 \\
Anacardiaceae & 2 & 2 & Total & & 43 \\
\hline
\end{tabular}




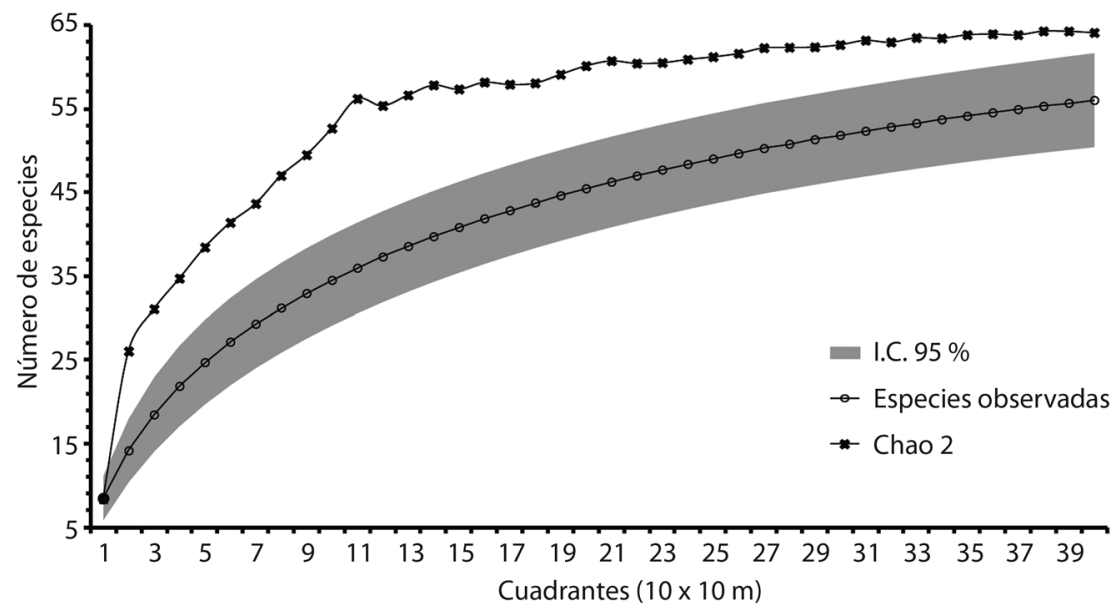

Fig. 2. Curva de acumulación de especies. I. C. = intervalo de confianza.

Fig. 2. Species accumulation curve. I. C. = confidence interval.

de diversidad de Shannon-Weaner obtenido fue de 2.79 con una equidad de 0.691 .

En cuanto a la composición de la flora leñosa, las familias con mayor número de especies fueron Fabaceae (15) y Rubiaceae (5), las demás estuvieron representadas por tres o menos especies (Cuadro 2). Los géneros más ricos fueron Randia con cuatro especies, Eugenia y Lonchocarpus con tres especies cada uno.

La distribución de los individuos en las clases diamétricas y de alturas presentó un arreglo de "J invertida" (Fig. 3), donde el número de individuos disminuye conforme se incrementan los valores en las clases, dominado con ello organismos de diámetros pequeños (1 y $10 \mathrm{~cm})$ y altura baja $(1.5-4.5 \mathrm{~m})$.

En referencia a la dominancia, es importante destacar que solamente cinco especies albergaron el $52.3 \%$ del índice VIR (Cuadro 3), estas fueron: Haematoxylum campechianum, Dalbergia glabra, Havardia albicans, Cameraria latifolia y Croton reflexifolius. El primer lugar de $H$. campechianum se debió a su alta dominancia relativa, como reflejo de la gran biomasa que aporta al ecosistema $\left(6.12 \mathrm{~m}^{2} / \mathrm{ha}\right)$, mientras que la liana leñosa $D$. glabra se ubicó

\section{CUADRO 3}

Análisis de valor de importancia relativa (VIR)

TABLE 3

Analysis of relative importance value (RIV)

\begin{tabular}{|c|c|c|c|c|c|c|c|}
\hline & $\begin{array}{c}\text { Densidad } \\
\text { absoluta }\end{array}$ & Área basal & $\begin{array}{c}\text { Densidad } \\
\text { relativa }\end{array}$ & $\begin{array}{l}\text { Frecuencia } \\
\text { relativa }\end{array}$ & $\begin{array}{l}\text { Dominancia } \\
\text { relativa }\end{array}$ & VIR & $\begin{array}{c}\text { VIR } \\
\text { acumulado }\end{array}$ \\
\hline Especies & \# ind. & $\mathrm{m}^{2} / \mathrm{ha}$ & $\%$ & $\%$ & $\%$ & $\%$ & $\%$ \\
\hline 1. Haematoxylum campechianum & 95 & 6.121 & 5.54 & 6.80 & 46.20 & 19.52 & 19.52 \\
\hline 2. Dalbergia glabra & 379 & 0.404 & 22.11 & 9.76 & 3.05 & 11.64 & 31.16 \\
\hline 3. Havardia albicans & 155 & 0.993 & 9.04 & 4.73 & 7.50 & 7.09 & 38.25 \\
\hline 4. Cameraria latifolia & 113 & 1.427 & 6.59 & 3.85 & 10.77 & 7.07 & 45.32 \\
\hline 5. Croton reflexifolius & 203 & 0.341 & 11.84 & 6.51 & 2.57 & 6.97 & 52.30 \\
\hline 6. Diospyros bumelioides & 194 & 0.661 & 11.32 & 4.44 & 4.99 & 6.92 & 59.21 \\
\hline 7. Zygia cognata & 105 & 0.878 & 6.13 & 5.33 & 6.63 & 6.03 & 65.24 \\
\hline 8. Terminalia buceras & 39 & 1.109 & 2.28 & 4.44 & 8.37 & 5.03 & 70.27 \\
\hline
\end{tabular}


CUADRO 3 (Continuación) / TABLE 3 (Continued)

\begin{tabular}{lccccccc} 
& $\begin{array}{c}\text { Densidad } \\
\text { absoluta }\end{array}$ & Área basal & $\begin{array}{c}\text { Densidad } \\
\text { relativa }\end{array}$ & $\begin{array}{c}\text { Frecuencia } \\
\text { relativa }\end{array}$ & $\begin{array}{c}\text { Dominancia } \\
\text { relativa }\end{array}$ & VIR & $\begin{array}{c}\text { VIR } \\
\text { acumulado }\end{array}$ \\
9. Mimosa bahamensis & 75 & 0.238 & 4.38 & 2.66 & 1.80 & 2.95 & 73.22 \\
10. Erythroxylum areolatum & 44 & 0.130 & 2.57 & 5.03 & 0.98 & 2.86 & 76.08 \\
11. Crescentia cujete & 28 & 0.151 & 1.63 & 3.85 & 1.14 & 2.21 & 78.28 \\
12. Bonellia albiflora & 26 & 0.017 & 1.52 & 3.55 & 0.13 & 1.73 & 80.02 \\
13. Randia aculeata & 18 & 0.008 & 1.05 & 3.25 & 0.06 & 1.45 & 81.47 \\
14. Coccoloba cozumelensis & 18 & 0.137 & 1.05 & 2.07 & 1.03 & 1.38 & 82.85 \\
15. Erythroxylum confusum & 35 & 0.070 & 2.04 & 1.48 & 0.53 & 1.35 & 84.20 \\
16. Eugenia oerstediana & 16 & 0.014 & 0.93 & 2.66 & 0.11 & 1.23 & 85.44 \\
17. Eugenia foetida & 23 & 0.020 & 1.34 & 2.07 & 0.15 & 1.19 & 86.62 \\
18. Hyperbaena winzerlingii & 10 & 0.067 & 0.58 & 1.78 & 0.51 & 0.96 & 87.58 \\
19. Capparis flexuosa & 10 & 0.009 & 0.58 & 1.78 & 0.06 & 0.81 & 88.39 \\
20. Lonchocarpus sp. & 11 & 0.016 & 0.64 & 1.48 & 0.12 & 0.75 & 89.13 \\
Total of other species (36) & 117 & 0.435 & 6.83 & 22.49 & 3.29 & 10.87 & 100.00 \\
Total & 1714 & 13.248 & 100.00 & 100.00 & 100.00 & 100.0 & - \\
\hline
\end{tabular}
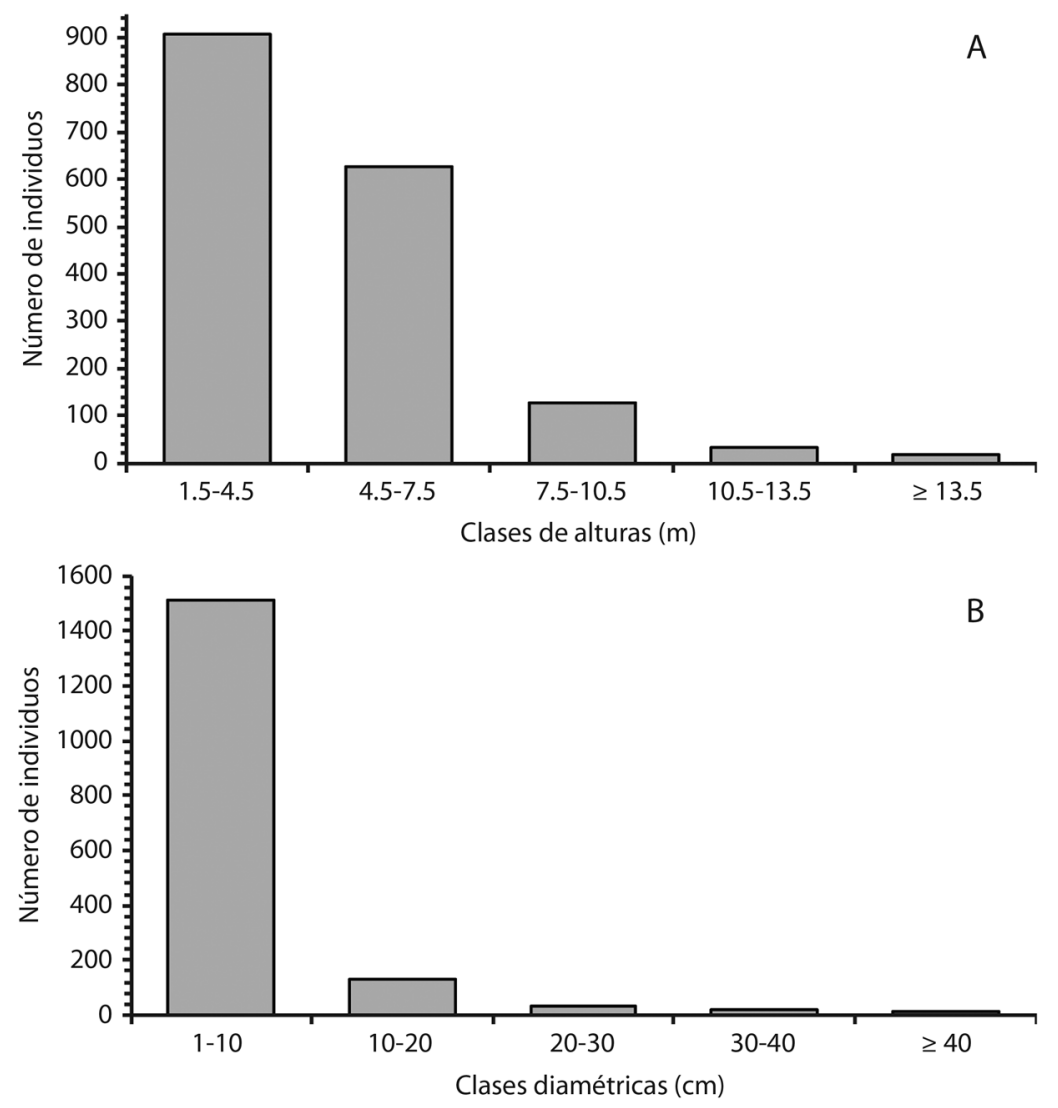

Fig. 3. Histogramas de distribución de individuos según sus diámetros (A) y sus alturas (B). Fig. 3. Histograms of individuals distribution according to diameters (A) and heights (B). 


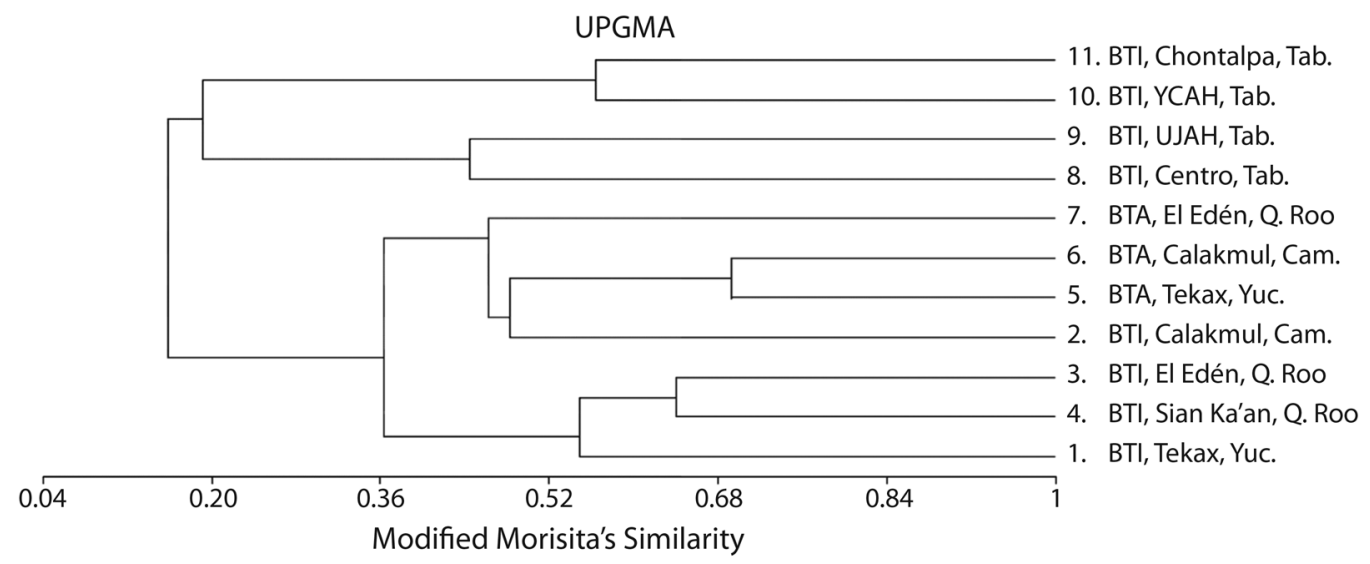

Fig. 4. Dendrograma de similitud florística. Los números de los sitios coinciden con los del Cuadro 1.

Fig. 4. Dendrogram of floristic similarity. Numbers of the sites according with those in Table 1.

en segundo lugar, al ser la especie con mayor abundancia (379 individuos).

Análisis de relaciones florísticas: La integración de la información florística de las BTI's y BTA's de la península de Yucatán y BTI's de Tabasco, resultó en una matriz compuesta por 192 géneros de plantas leñosas.

Del análisis de similitud se obtuvo un dendrograma (Fig. 4) cuya topología identifica la formación de dos grandes grupos (con $16 \%$ de similitud), el primero integrado por los BTI's de Tabasco (19\% de similitud) y el segundo por los BTA's y BTI's de la península de Yucatán (36\% de similitud). Dentro de este último se nota la diferenciación de dos subgrupos, el primero integrado por todos los BTA's y el BTI de Calakmul (46\% de similitud) y el segundo por los restantes 3 BTI's (55\% de similitud) que incluye a nuestra zona de estudio. Las similitudes máximas entre sitios se presentaron entre los BTA's de Calakmul y Tekax (69\% de similitud; $149 \mathrm{~km}$ de distancia), y los BTI's de El Edén y Sian Ka'an (64\% de similitud; 167 km de distancia). No se observó una correlación significativa entre la matriz de distancias geográficas y la matriz de similitud $(r=-0.098, p>0.05)$, lo que sugiere que sitios geográficamente más cercanos no fueron más similares entre sí.

\section{DISCUSIÓN}

En el presente trabajo contribuimos al conocimiento de la diversidad, estructura y afinidades florísticas de los BTI's de la península de Yucatán.

Los resultados de riqueza y diversidad obtenidos son inferiores a los reportados para BTA's de la región de estudio (Zamora-Crescencio et al., 2008; Gutiérrez-Báez et al., 2012). Por el contrario, son similares a los de otros bosques temporalmente inundables del sureste de México (Olmsted \& Durán, 1986; Díaz-Gallegos et al., 2002; Hernández-Ramírez \& García-Méndez, 2015; Maldonado-Sánchez et al., 2016). La baja riqueza y diversidad de los BTI's en la región es atribuible a la anoxia por inundación durante la época de lluvias, y a la contracción del suelo en época de seca, factores selectivos para el establecimiento y supervivencia de plántulas (Parolin et al., 2004; Cortés-Castelán \& Islebe, 2005; Moreno-Casasola \& Infante-Mata, 2009).

En cuanto a la composición florística, la riqueza de especies de las familias Fabaceae y Rubiaceae concuerda con la tendencia reportada para los bosques neotropicales (Pennington, Lewis \& Ratter, 2006). La flora leñosa registrada denota una afinidad neotropical típica de hábitats inundables, ya que comparten 
los géneros de mayor dominancia (e. g. Byrsonima, Cameraria, Crescentia, Dalbergia, Haematoxylum y Terminalia) con sabanas y bosques inundables de tierras bajas en Centroamérica y Brasil (Ellison, 2004; Junk, Piedade, Wittmann, Schöngart, \& Parolin, 2010; Goodwin et al., 2013).

La estructura del BTI estudiado se caracterizó por la dominancia de las clases menores de diámetros y de alturas, lo cual es similar a lo reportado para la mayoría de bosques tropicales del sureste de México y Centroamérica (Pennington et al., 2006). Sin embargo, una característica estructural relevante es la alta densidad de lianas, lo cual denota el óptimo estado de conservación de la comunidad (López-Martínez et al., 2013).

El registro de una alta dominancia de las especies concuerda con lo reportado por otros estudios de BTI's del sureste de México (Lot \& Novelo, 1990; Olmsted, 1993; Infante-Mata, Moreno-Casasola, \& Madero-Vega, 2014;Maldonado-Sánchez et al., 2016). Esta dominancia puede variar entre comunidades según la intensidad y duración de la inundación (Olmsted \& Durán, 1986; López \& Kursar, 2003; Cortés-Castelán \& Islebe, 2005; Kurzatkowski et al., 2015) o de la capacidad de las especies de los BTA's adyacentes para generar ecotipos adaptables a la inundación que contribuyan a aumentar la riqueza florística y equidad del ensamblaje de especies de los BTI's (Ellison, 2004; Flinn et al., 2008).

Según nuestro análisis de VIR, la dominancia de las especies puede expresarse de dos maneras en el ensamblaje de los BTI's: 1) por la biomasa que aportan a la comunidad, como Haematoxylum campechianum, o 2) a través de una alta abundancia como Dalbergia glabra. Debido a que ambas especies han sido reconocidas como típicas de los bosques inundables de la península de Yucatán (Miranda, 1978; Lot \& Novelo, 1990; Olmsted, 1993; Rzedowski, 2006), posiblemente posean adaptaciones fisiológicas como mecanismos de regulación fotosintética, metabólica o de eficiencia en la oxigenación de tejidos (López, 2009; Parolin et al., 2004; Kurzatkowski et al., 2015).
En un contexto regional, encontramos que los BTI's son más similares a sus BTA's adyacentes (de la península de Yucatán) que a los BTI's de otras regiones (estado de Tabasco). Estas diferencias radican en que los BTI's están florísticamente integrados por especies "típicas" y "ecotipos" de BTA's (Wittmann et al., 2006). Por lo que, al comparar BTI's y BTA's de la misma región como lo hicimos con los de la península de Yucatán, es posible reconocer que los elementos típicos de BTI's (e. g. Cameraria latifolia, Crescentia cujete, Dalbergia glabra, Haematoxylum campechianum, Terminalia buceras, entre otros) son responsables de las diferencias florísticas entre ambas comunidades vegetales. Mientras, el número de ecotipos compartidos con los BTA's (e. g. Coccoloba cozumelensis, Croton reflexifolius, Havardia albicans, Mimosa bahamensis, entre otros) aumenta su similitud (López \& Kursar, 2003; Kurtz, Gomes \& Scarano, 2013), que según nuestros análisis no está relacionada con la distancia geográfica. En el análisis de relaciones florísticas, únicamente el BTI de Calakmul se agrupó con los BTA's, posiblemente por la presencia de menor estrés natural por inundación. Otro factor posible es que se encuentra adyacente a un BTA de tipo perennifolio, esto es importante, ya que sus ecotipos compartidos poseen mayor tolerancia a la inundación que los de BTA's caducifolios (Cortés-Castelán $\&$ Islebe, 2005).

Al comparar BTI's de diferentes regiones como lo hicimos con los del estado de Tabasco (Morales-Ramos, 2008; Vázquez-Negrín, López-Pérez, Montalvo-Urgel, Méndez Sánchez, \& Castillo Acosta, 2010; MaldonadoSánchez et al., 2016), su similitud florística es menor, ya que aunque comparten elementos (e. g. Bonellia macrocarpa, Haematoxylum campechianum, Zygia cognata, etc.), estos bosques poseen diferentes especies dominantes (e. g. Bravaisia integérrima, Spondias mombin, Tabernaemontana alba, etc.) y diferentes ecotipos de BTA's adyacentes que han logrado establecerse en ellos (e. g. Acacia cornigera, Bursera simaruba, Crecropia obtusifolia, entre otras). Lo que enfatiza la necesidad de generar 
estrategias de conservación para los BTI's de la península de Yucatán de manera independiente a otras regiones del sureste de México.

En conclusión, en el presente estudio encontramos que el BTI en la región estudiada posee una diversidad similar a otros BTI's del sureste de México, una composición florística diferente a BTA's adyacentes y una estructura que sugiere un adecuado estado de conservación, características que denotan su importancia para la conservación de la flora regional. Así mismo, evidenciamos desde una perspectiva florística, que BTI's de una misma región pueden ser más similares a sus BTA's adyacentes que a BTI's de otras regiones geográficas. La información generada es prioritaria para el establecimiento de bases sólidas para la conservación y el mantenimiento los BTI's. Estudios a futuro deben abordar a fina escala las diferencias entre BTI's y BTA's en sitios contiguos y correlacionar sus diferencias con factores físicos como el relieve, composición del suelo y grado de inundación.

\section{AGRADECIMIENTOS}

Los autores agradecemos a los revisores anónimos por el tiempo y trabajo dedicado al presente manuscrito. Al personal de los herbarios UADY y CICY por su apoyo en la consulta de material de herbario. A Leonardo Ék Rodríguez, Miguel Flandes, Jorge de la Cruz y Kevin Baaz por su apoyo en campo. A Sandra López Manzanero por sus cometarios al presente escrito. A Juan Ornelas por su apoyo con la traducción del resumen al idioma inglés.

\section{RESUMEN}

Los bosques temporalmente inundables (BTI's) se encuentran entre las comunidades arbóreas más amenazadas del sureste de México y de manera contradictoria entre las menos conocidas en términos de su diversidad. Por lo que el objetivo del presente estudio fue caracterizar la riqueza, diversidad (Shannon-Wiener) y dominancia (valor de importancia relativo; VIR) del componente leñoso de un BTI de la península de Yucatán, así como evaluar sus afinidades florísticas con otros BTI's y bosques de tierras altas (BTA's) del sureste de México. Se utilizó un método de muestreo basado 10 cuadrantes de $10 \times 20$ m (2 000 $\mathrm{m}^{2}$ ), donde se registraron todos los individuos leñosos con diámetro $\geq 1 \mathrm{~cm}$ y altura $\geq 1.5 \mathrm{~m}$. Se usó un análisis de similitud florística que incluyó 192 géneros de plantas leñosas presentes en BTI's y BTA's de la península de Yucatán y BTI's Tabasco. Se realizó una prueba de correlación de Mantel para determinar si la distancia geográfica posee efecto sobre su similitud florística entre BTI's y BTA's de la península de Yucatán. El BTI estudiado se caracterizó por una baja diversidad (2.79) y riqueza de especies (56), así como por una alta dominancia $(52.3 \%$ del VIR en cinco especies). Especies dominantes como Haematoxylum campechianum, Dalbergia glabra, Cameraria latifolia, Byrsonima crassifolia, Crescentia cujete y Terminalia buceras denotan una afinidad florística neotropical típica de hábitats inundables. La estructura de la comunidad se caracterizó por presentar un patrón de "J" invertida para la distribución de los individuos en las clases diamétricas y de alturas. La alta densidad de lianas leñosas principalmente de Dalbergia glabra (22\% de los individuos registrados) sugiere un óptimo estado de conservación para el área de estudio. El análisis de relaciones florísticas reveló que en su mayoría BTI's de la península de Yucatán se agrupan con alta similitud (55 \%) independientemente de los BTA's adyacentes, sin embargo, guardan mayor similitud con ellos (36\% de similitud) que con BTI's de otras regiones (16\% de similitud). Según la prueba de Mantel, la similitud entre sitios no se encuentra influenciada por su proximidad geográfica $(r=-0.098, p>0.05)$. Esto enfatiza la necesidad de generar estrategias de conservación para los BTI's de la península de Yucatán de manera independiente de otras regiones del sureste de México. Nuestros resultados sugieren que el BTI de la región estudiada posee una diversidad similar a otros BTI's del sureste de México, una composición florística diferente de BTA's adyacentes y una estructura con óptimo estado de conservación, características que indican su importancia para la conservación de la flora regional. Son necesarios estudios ecológicos adicionales para generar bases sólidas para el manejo y conservación de los BTI's.

Palabras clave: selva baja inundable, península de Yucatán, humedal, diversidad vegetal, relaciones florísticas, Haematoxylum campechianum.

\section{REFERENCIAS}

Acosta, S. (2004). Afinidades de la flora genérica de algunos bosques mesófilos de montaña del nordeste, centro y sur de México: un enfoque fenético. Anales del Instituto de Biología Serie Botánica, 75(1), 61-72.

Bautista, F., \& Zinck, J. A. (2010). Construction of an Yucatec Maya soil classification and comparison with the WRB framework. Journal of Ethnobiology and Ethnomedicine, 6, 7. http://doi. org/10.1186/1746-4269-6-7 
Berlanga-Robles, C. A., Ruiz-Luna, A., \& de la Lanza Espino, G. (2008). Esquema de clasificación de los humedales de México. Investigaciones Geográficas, 66, 25-46. http://doi.org/10.14350/rig.17980

Briceño-Méndez, M., Reyna-Hurtado, R., Calmé, S., \& García-Gil, G. (2014). Preferencias de hábitat y abundancia relativa de Tayassu pecari en un área con cacería en la región de Calakmul, Campeche, México. Revista Mexicana de Biodiversidad, 85(1), 242-250. http://doi.org/10.7550/rmb.31937

Chan-Dzul, A. M. (2010). Diversidad florística y funcional a través de una cronosecuencia de la selva mediana subperennifolia en la zona de influencia de la Reserva de la Biosfera Calakmul, Campeche, México (Tesis de maestría). Centro Agronómico Tropical de Investigación y Enseñanza (CATIE), Costa Rica.

Chase, M. W., \& Reveal, J. L. (2009). A phylogenetic classification of the land plants to accompany APG III. Botanical Journal of the Linnean Society, 161, 122-127. http://doi.org/10.1111/j.1095-8339.2009.01002.x

Colwell, R. K. (2013). EstimateS: Statistical estimation of species richness and shared species from samples. Retrieved from http://purl.oclc.org/estimates

Cortés-Castelán, J. C. \& Islebe, G. A. (2005). Influencia de factores ambientales en la distribución de especies arbóreas en las selvas del sureste de México. Revista de Biología Tropical, 53(1-2), 115-133. http://doi. org/10.15517/rbt.v53i1-2.14373

Díaz-Gallegos, J., Castillo-Acosta, O., \& García-Gil, G. (2002). Distribución espacial y estructura arbórea de la selva baja subperennifolia en un ejido de la Reserva de la Biosfera Calakmul, Campeche, México. Universidad y Ciencia, 18(35), 11-28.

Duch-Gary, J. (1988). La conformación territorial del estado de Yucatán: los componentes del medio físico. México: Universidad Autónoma Chapingo.

Duch-Gary, J. (1989). Los bajos inundables (ak'alches) de la Península de Yucatán: las expectativas de una evaluación ambiental referida a su aprovechamiento. México: Universidad Autónoma Chapingo.

Ellison, A. M. (2004). Wetlands of central America. Wetlands Ecology and Management, 12(1), 3-55. http:// doi.org/10.1023/B:WETL.0000016809.95746.b1

Finlayson, C. M., \& van der Valk, A. G. (1995). Wetland classification and inventory: A summary. Vegetatio, 118(1-2), 185-192. http://doi.org/10.1007/ BF00045199

Finlayson, C. M., \& van der Valk, A. G. (2012). Classification and Inventory of the World's Wetlands. Springer Science \& Business Media.

Flinn, K. M., Lechowicz, M. J., \& Waterway, M. J. (2008). Plant species diversity and composition of wetlands within an upland forest. American Journal of Botany, 95(10), 1216-1224. http://doi.org/10.3732/ ajb.0800098

Flores, J. S. \& Espejel, I. (1994). Tipos de vegetación de la Península de Yucatán. Yucatán, México: Universidad Autónoma de Yucatán, Dirección General de Extensión.

Gentry, A. H. (1982). Patterns of Neotropical plant species diversity (Vol. 15). Missouri: Springer US.

Giraldo-Cañas, D. (2001). Relaciones fitogeográficas de las sierras y afloramientos rocosos de la Guayana colombiana: un estudio preliminar. Revista Chilena de Historia Natural, 74(2), 353-364. http://doi. org/10.4067/S0716-078X2001000200012

Goodwin, Z. A., Harris, D. J., Bridgewater, S. G. M., López, G. N., Haston, E. M., Cameron, I. D., ... Stuart, N. (2013). A checklist of the vascular plants of the lowland savannas and associated wetlands of Belize, Central America. Phytotaxa, 101(1), 1-119. http://doi.org/10.11646/phytotaxa.101.1.1

Gutiérrez-Báez, C., Ortiz-Díaz, J., Flores-Guido, J. S., \& Zamora-Crescencio, P. (2012). Diversidad, estructura y composición de las especies leñosas de la selva mediana subcaducifolia del punto de unión territorial (PUT) de Yucatán, México. Polibotánica, 33(14052768), 151-174.

Hernández-Ramírez, A. M. \& García-Méndez, S. (2015). Diversidad, estructura y regeneración de la selva tropical estacionalmente seca de la Peninsula de Yucatan, México. Revista de Biología Tropical, 63(3), 603-616.

INEGI. (2009). Prontuario de información geográfica municipal de los Estados Unidos Mexicanos: Hoja Tekax, Yucatán. México: INEGI.

Infante-Mata, D., Moreno-Casasola, P., \& Madero-Vega, C. (2014). ¿Pachira aquatica, un indicador del límite del manglar? Revista Mexicana de Biodiversidad, 85(1), 143-160. http://doi.org/10.7550/rmb.32656

ITHAKA. (2015). Global Plants. Retrieved from http:// plants.jstor.org/

Junk, W. J., Piedade, M. T. F., Wittmann, F., Schöngart, J., \& Parolin, P. (2010). Amazonian Floodplain Forests: Ecophysiology, Biodiversity and Sustainable Management. Ecological Studies (Vol. 210). Cambrige: Cambrige University Press. http://doi.org/10.1017/ CBO9781107415324.004

Kurtz, B. C., Gomes, J. C., \& Scarano, F. R. (2013). Structure and phytogeographic relationships of swamp forests of Southeast Brazil. Acta Botanica Brasilica, 27(4), 647-660. http://doi.org/10.1590/ S0102-33062013000400002 
Kurzatkowski, D., Leuschner, C., \& Homeier, J. (2015). Effects of flooding on trees in the semi-deciduous transition forests of the Araguaia floodplain, Brazil. Acta Oecologica, 69, 21-30. http://doi.org/10.1016/j. actao.2015.08.002

López, O. R. (2009). Fisiología y ecología de comunidades arbóreas en hábitats inundables. Acta Biológica Panamensis, 1, 68-86.

López, O. R., \& Kursar, T. A. (2003). Does flood tolerance explain tree species distribution in tropical seasonally flooded habitats? Oecologia, 136, 193-204. http://doi. org/10.1007/s00442-003-1259-7

López-Martínez, J. O., Sanaphre-Villanueva, L., Dupuy, J. M., Hernández-Stefanoni, J. L., Meave, J. A., \& Gallardo-Cruz, J. A. (2013). $\beta$-Diversity of Functional Groups of Woody Plants in a Tropical Dry Forest in Yucatan. PLoS ONE, 8(9). http://doi.org/10.1371/ journal.pone. 0073660

Lot, H. A., \& Novelo, A. (1990). Forested Wetlands of Mexico. In A. E. Lugo, M. Brison, \& S. Brown (Eds.), Forested Wetlands of the World (pp. 287-298). Amsterdam, The Netherlands: Elsevier.

Lundell, C. L. (1934). Preliminary sketch of the phytogeography of the Yucatan Peninsula. Washington, D.C.: Carnegie Institute of Washington.

Magurran, A. E. (1988). Ecological diversity and its measurement (Vol. 179). New Yersey, USA: Princeton University Press.

Maldonado-Sánchez, E. A., Ochoa-Gaona, S., RamosReyes, R., Guadarrama-Olivera, M., González-Valdivia, N., \& de Jong, B. H. J. (2016). La selva inundable de canacoite en Tabasco, México, una comunidad vegetal amenazada. Acta Botánica Mexicana, 115, 75-101.

Migeot, J., \& Imbert, D. (2012). Phenology and production of litter in a Pterocarpus officinalis (Jacq.) swamp forest of Guadeloupe (Lesser Antilles). Aquatic Botany, 101, 18-27. http://doi.org/10.1016/j. aquabot.2012.03.012

Miranda, F. (1958). Estudios acerca de la vegetación. In E Beltrán (Ed.), Los recursos naturales del sureste y su aprovechamiento (pp. 215-271). México, D.F.: Ediciones del Instituto Mexicano de Recursos Naturales Renovables.

Miranda, F. (1978). Vegetación de la península yucateca; rasgos fisiográficos, la vegetación. México: Chapingo SARH.

Miranda, F., \& Hernández, X. E. (1963). Los tipos de vegetación de México y su clasificación. Boletín de la Sociedad Mexicana de México (Vol. 28). México:
Colegio de Postgraduados, Secretaría de Agricultura y Recursos Hidráulicos.

Morales-Ramos, S. del C. (2008). Estructura y composición floristical del Tintal (Haematoxylum campechianum L.) en tres comunidades de Tabasco. México: Universidad Juárez Autónoma de Tabasco.

Moreno, C. E. \& Pineda, E. (2011). Reanálisis de la diversidad alfa : alternativas para interpretar y comparar información sobre comunidades ecológicas. Revista Mexicana de Biodiversidad, 82, 1249-1261. http:// doi.org/10.7550/rmb.28802

Moreno-Casasola, P., Infante-Mata, D., \& López-Rosas, H. (2012). Tropical freshwater swamps and marshes. In D. Batzer \& A. Baldwin (Eds.), Wetland habitats of North America: ecology and conservation concerns (pp. 267-282). Berkeley: University of California Press.

Moreno-Casasola, P., \& Infante-Mata, D. M. (2009). Manglares y selvas inundables. Xalapa, México: Instituto de Ecología A. C., CONAFOR \& OIMT.

Mueller-Dombois, D. \& Ellenberg, H. (1974). Aims and methods of vegetation ecology. USA: John Wiley \& Sons.

Olmsted, I. (1993). Wetlands of Mexico. In D. F. Whigham, D. Dykyjová, \& S. Hejny (Eds.), Wetlands of the world: Inventory, ecology and management (Volume I, pp. 637-677). Dordrecht, The Netherlands: Kluwer Academic Publishers. http://doi. org/10.1007/978-94-015-8212-4_13

Olmsted, I. \& Durán, R. (1986). Aspectos ecológicos de la selva baja inundable de la Reserva de la Biosfera de Sian Ka'an, Quintana Roo, México. Biotica, 11, 151-179.

Palacio-Aponte, A. G., Noriega-Trejo, R., \& Zamora-Crescencio, P. (2002). Caracterizacion fisico-geografica del paisaje conocido como "bajos inundables". El caso del area Natural Protegida Balamkin, Campeche. Investigaciones Geograficas, 49, 57-73. http:// doi.org/10.14350/rig.30446

Parolin, P., De Simone, O., Haase, K., \& Waldhoff, D. (2004). Central Amazonian floodplain forests: tree adaptations in a pulsing system. The Botanical Review, 70(3), 357-380. http://doi. org/10.1663/0006-8101(2004)070[0357:CAFFTA] 2.0.CO;2

Pennington, R. T., Lewis, G. P., \& Ratter, J. A. (2006). Neotropical savannas and seasonally dry forests: plant diversity, biogeography, and conservation. Economic Botany, 61(3), 311-312. http://doi. org/10.1663/0013-0001(2007)61[311b:NSASDF] 2.0.CO;2 
Rzedowski, J. (2006). Vegetacion de Mexico (I edicion digital). México: Comisión Nacional para el Conocimiento y Uso de la Biodiversidad.

Sánchez-Sánchez, O., Mendizábal-Hernández, L. C., \& Calmé, S. (2006). Recuperación foliar en un acahual después del paso del huracán Wilma por la reserva ecológica el Eden, Quintana Roo. Foresta Veracruzana, 8(1), 37-42.

Schultz, G. P. (2005). Vascular flora of the El Edén Ecological Reserve, Quintana Roo, Mexico. Journal of the Torrey Botanical Society, 132(2), 311-322. http://doi. org /10.3159/1095-5674(2005)132[311:VFOTEE]2.0

Scott, D. A., \& Carbonell, M. (1986). A directory of neotropical wetlands. Cambridge: IUCN, IWRB.

Scott, D. A., \& Jones, T. A. (1995). Classification and Inventory of Wetlands : A Global Overview. Vegetatio, 118(1), 3-16. http://doi.org/10.1007/BF00045186
Thiers, B. (2012). Index Herbariorum: A global directory of public herbaria and associated staff. USA: New York Botanical Garden's Virtual Herbarium. GEN.

Vázquez-Negrín, I., López-Pérez, D., Montalvo-Urgel, H. E., Méndez Sánchez, C. A., \& Castillo Acosta, O. (2010). Estructura y composición florística de vegetación inundable en la División Académica de Ciencias Biológicas, Villahermosa, Tabasco. Kuxulkab', 17, 21-30.

Wittmann, F., Schöngart, J., Montero, J. C., Motzer, T., Junk, W. J., Piedade, M. T. F., ... Worbes, M. (2006). Tree species composition and diversity gradients in white-water forests across the Amazon Basin. Journal of Biogeography, 33(8), 1334-1347. doi. org/10.1111/j.1365-2699.2006.01495.x 1

Zamora-Crescencio, P., García-Gil, G., Flores, J. S., \& Ortiz, J. J. (2008). Estructura y composición florística de la selva sediana subcaducifolia en el sur del estado de Yucatán, México. Polibotánica, 26, 39-66. 